\title{
Simulation Study on Blast Resistance Test of Blast Wall Based on LS-DYNA Coupling Method
}

\author{
Zhu Gan ", Ding Jianguo \\ College of Science, Nanjing University of Scinece and Technology, Nanjing, China \\ Email address: \\ 783148731@qq.com (Zhu Gan), nustdjg@yahoo.com.cn (Ding Jianguo) \\ ${ }^{*}$ Corresponding author
}

To cite this article:

Zhu Gan, Ding Jianguo. Simulation Study on Blast Resistance Test of Blast Wall Based on LS-DYNA Coupling Method. Science Discovery. Vol. 9, No. 3, 2021, pp. 114-120. doi: 10.11648/j.sd.20210903.16

Received: April 14, 2021; Accepted: May 10, 2021; Published: May 24, 2021

\begin{abstract}
In order to study the pressure change with time at the blast face of the blast resistant wall when the shock wave acts on the blast resistant wall after the explosion of TNT explosive, the equivalent model of the blast resistant wall is designed based on the similarity theory, and the TNT near ground explosion test is carried out. During the test, the pressure change data of the design measuring point on the blast resistant wall with time are collected by high-speed data acquisition instrument and pressure sensor, The time history curve of shock wave overpressure was obtained by processing the data. Then, LS-DYNA finite element analysis software is used to establish a separate finite element model of the blast wall. At the same time, the relevant parameters of air, explosive, concrete and ground in the explosion field are set. The numerical simulation of the explosion field is carried out, and the overpressure time history curve at the measuring point is extracted by the finite element analysis post-processing software. Compared with the numerical simulation results, it is found that the numerical simulation results are in good agreement with the experimental data, which indicates that the selection and setting of parameters in the simulation calculation are reasonable. The model and algorithm provide an effective means for the numerical simulation of blast wall in the explosion field.
\end{abstract}

Keywords: TNT, Explosion Shock Wave, Blast Wall, LS-DYNA

\section{基于LS-DYNA耦合算法的抗爆墙抗爆试验仿真研究}

朱淦”，丁建国

南京理工大学理学院, 南京, 中国

邮箱

783148731@qq.com(朱淦), nustdjg@yahoo.com.cn ( 丁建国)

摘要: 为了研究TNT炸药爆炸后冲击波作用到抗爆墙时, 抗爆墙迎爆面处压力随时间变化情况, 依托相似理论设计了 抗爆墙等缩模型并对其进行了 TNT近地面爆炸试验, 试验过程中, 通过高速数据采集仪和压力传感器采集抗爆墙上设 计测点的压力随时间变化数据, 处理数据得到冲击波超压时程曲线。随后, 应用LS-DYNA有限元分析软件对设计的抗 爆墙建立了抗爆墙的分离式有限元模型, 同时设置了爆炸场中空气、炸药、混凝土及地面的相关参数, 对爆炸场进行 数值仿真计算, 通过有限元分析后处理软件提取测点处的超压时程曲线。试验结果与数值仿真结果对比后发现, 数值 模拟的结果与试验数据吻合度较高, 表明仿真计算中参数的选取及设置具有合理性, 该模型和算法为抗爆墙在爆炸场 中的数值模拟提供了一种有效手段。

关键词: TNT, 爆炸冲击波, 抗爆墙, LS-DYNA 


\section{1. 引言}

研究抗爆墙迎爆面处的超压峰值对抗爆墙设计具有 重要的作用, 特别是研究近地面爆炸对抗爆墙的冲击作用, 更具有着现实意义。Henrych、张守中、 $\mathrm{Wu}$ 、仲倩等都对 TNT爆炸后地面反射超压进行了相关的研究, 并给出了相 关的经验公式 [1-4]。本文设计并完成了 TNT爆炸作用下的 抗爆墙抗爆试验, 采集了在设计工况下的抗爆墙迎爆面处 压力变化情况, 对其进行滤波处理后, 找出了冲击波在抗 爆墙处的变化规律。

梁利平等采用AUTODYN对TNT炸药起爆后冲击波 遇到挡墙时的传播规律进行模拟, 分析冲击波遇到挡墙后 产生的反射超压及爆炸冲击波的环流汇聚效应[5]。刘伟等 为研究近地面化学爆炸冲击波传播过程变化特性 [6], 在试 验场中进行了不同当量的TNT近地面爆炸试验, 并利用有 限元分析软件模拟了其中 2 种工况下的爆炸冲击波传播过 程。基于对抗爆墙在爆炸场中的耦合模拟的研究不够充分 这一现状，本文采用LS-DYNA软件对抗爆墙在近地面 TNT爆炸场的变化情况进行了数值模拟, 与试验测试结果
进行了比较, 为爆炸后冲击波与结构的耦合建模提供了相 关的参数参考 $[7-8]$ 。

\section{2. 近地面爆炸试验}

\section{1. 试验模型}

为获取钢筋混凝土抗爆墙在 TNT 爆炸作用下抗爆墙 迎爆面的超压峰值变化情况, 依托相似理论设计并制作了 钢筋混凝土抗爆墙缩尺模型, 并对抗爆墙模型进行抗爆试 验[9]。

抗爆墙原型为某工厂围护抗爆墙, 原型尺寸长度 $6 \mathrm{~m}$, 高度 $2.1 \mathrm{~m}$, 厚度 $0.6 \mathrm{~m}$ 。抗爆墙混凝土强度标号为 $\mathrm{C} 50$, 横 向配筋率 $0.8 \%$, 纵向配筋率 $1.7 \%$ 。综合考虑试验成本、施 工作业难易程度及模拟结果推导偏差程度等因素后, 确定 本试验抗爆墙按相似理论进行 $1: 3$ 的比例进行缩小, 模型 尺寸长度 $2 \mathrm{~m}$, 高度 $0.7 \mathrm{~m}$, 厚度 $0.2 \mathrm{~m}$, 混凝土强度标号为 C50。混凝土配合比如表1所示。

表1 混凝土配合比。

\begin{tabular}{lllllll}
\hline 水泥 & 粉煤灰 & 矿粉 & 砂 & 石 & 外加剂 & 水胶比 \\
\hline 0.62 & 0.18 & 0.2 & 1.37 & 2.21 & 0.014 & 0.33 \\
\hline
\end{tabular}

依据配筋率不变原则, 模型配筋率与原型相同。模型具体结构尺寸及配筋图如图1所示。
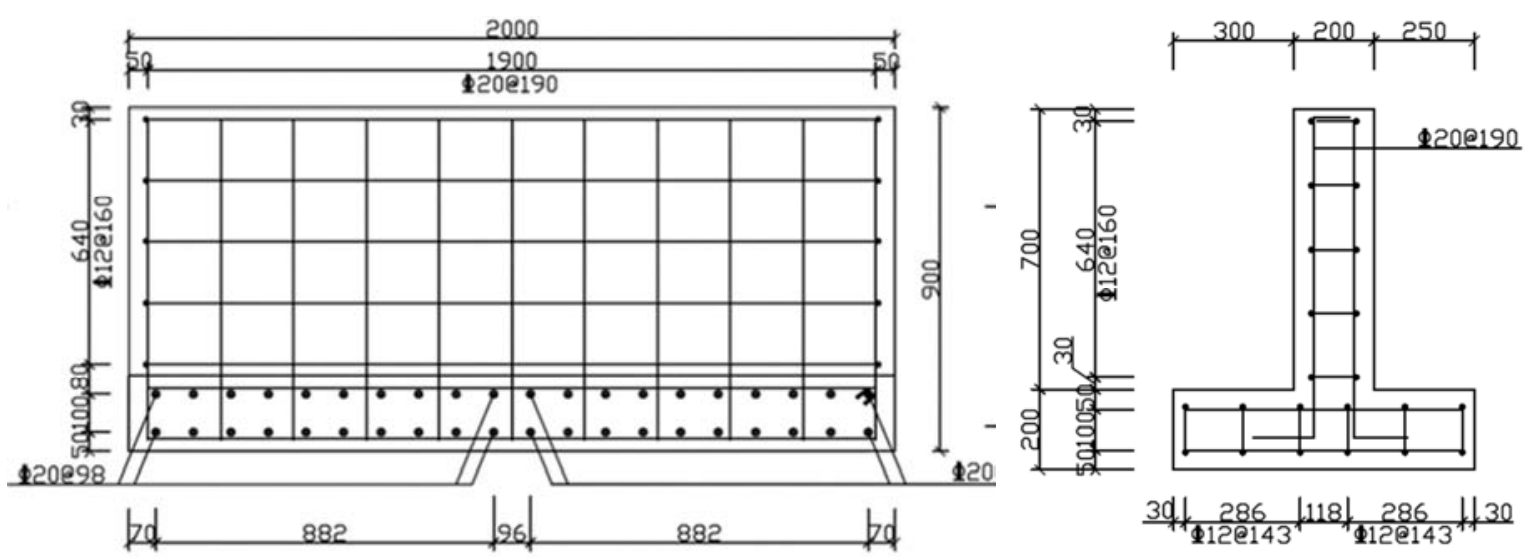

图1 抗爆墙模型配筋图。

\section{2. 试验工况}

本次试验选用TNT作为爆炸源, 具体工况安排如表2所示。

表2 试验工况。

\begin{tabular}{|c|c|c|c|c|}
\hline 药量 $W / \mathbf{k g}$ & 爆心距 $R / \mathbf{m}$ & 比距离 $Z$ & 超压 $\Delta \boldsymbol{P}_{c m} / \mathrm{Mpa}$ & 等效作用时间 $t_{0} / \mathrm{ms}$ \\
\hline 1.0 & 3.0 & 3.000 & 0.089 & 0.960 \\
\hline
\end{tabular}

为满足近地面爆炸的相关要求, TNT炸药放置于距离地面 $0.47 \mathrm{~m}$ 处。同时为保证超压测试点的有效性, TNT药柱放 置于抗爆墙模型中轴线上, TNT爆炸点具体布置情况如图2所示。 


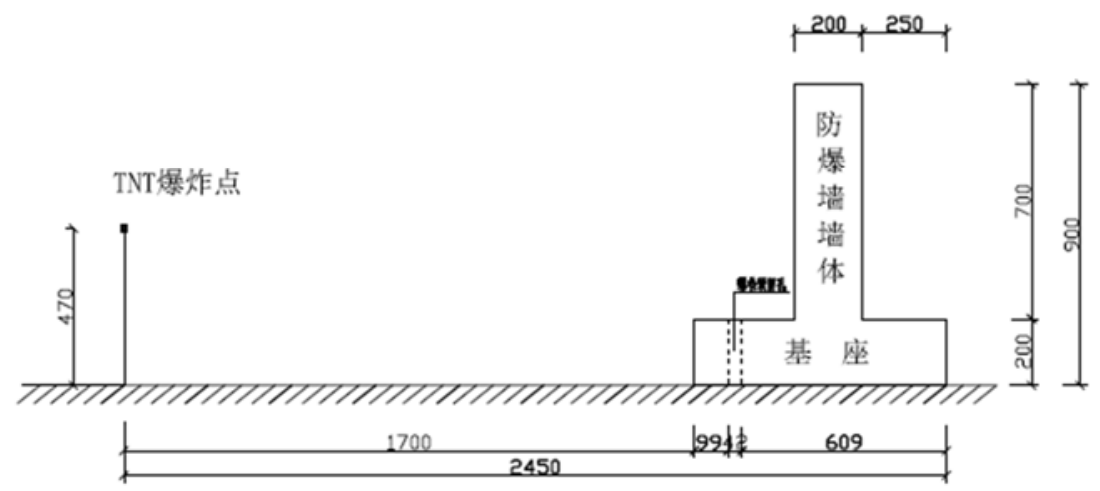

图2 试验布置情况。

\section{3. 试验测量}

数据采集软件使用DH8302高速数采仪配套的数据采 集分析软件，该软件由东华测试有限公司随硬件配发，内 含DHDAS 5920动态信号采集分析系统（与DH5920型高 速数据记录仪配套使用)，动态信号采集分析系统及软件 平台信号测试分析系统。
压力传感器选用试验室配备的CYG4100型压阻式高 频压力传感器, 该传感器内置压力放大器, 内置传感器与 信号调理器。为了防止电磁波的干扰影响试验测量数据的 采集, 使用抗干扰能力较强的四芯式电缆线用于连接传感 器与接收器。压力传感器的规格参数如表 3 所示。

表3 压力传感器的规格参数。

\begin{tabular}{lllllll}
\hline 型号 & 量程/MPa & 准确度等级 & 补偿温度范围 $/{ }^{\circ} \mathrm{C}$ & 使用温度范围 $/{ }^{\circ} \mathrm{C}$ & 频率 $/ \mathbf{K H z}$ & 灵敏度以及零位温度系数 $/{ }^{\circ} \mathrm{C} \cdot \mathbf{F} \cdot \mathbf{S}$ \\
\hline CYG4100 & $0 \sim 10$ & 0.25 级 & $0 \sim 600$ & $-10 \sim 700$ & $\geq 5000$ & $\leq 1.0 \times 10^{-4}$ \\
\hline
\end{tabular}

抗爆墙模型共设置 4 个超压测点, 均布置在抗爆墙迎 爆面上, 具体布置图如图3所示。
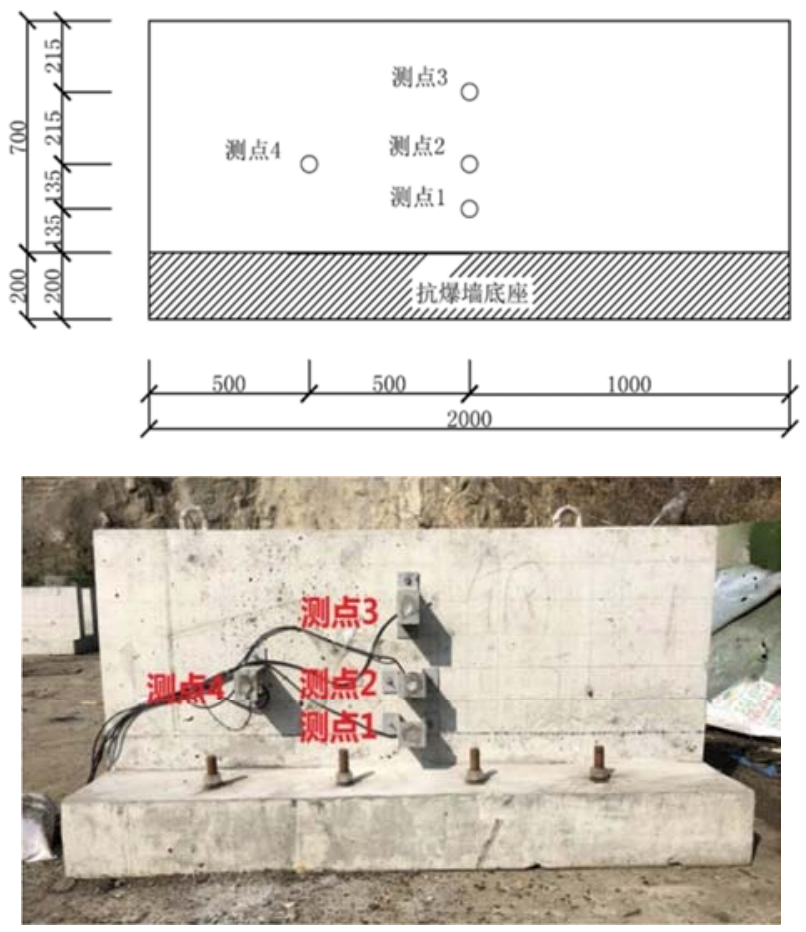

图3 超压测点布置图。

\section{4. 试验结果}

提取数据采集器中 3 \#测点的压力时程曲线, 经过截断、 平移、化零处理后可得如图4a)所示压力时程曲线图。对
其进行滤波处理, 可得一个平稳的曲线, 如图4b)内红色 曲线所示。

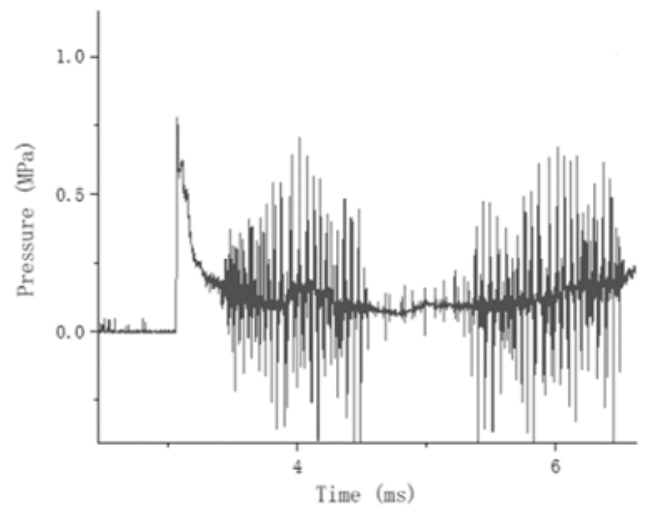

a）滤波前压力时程曲线图

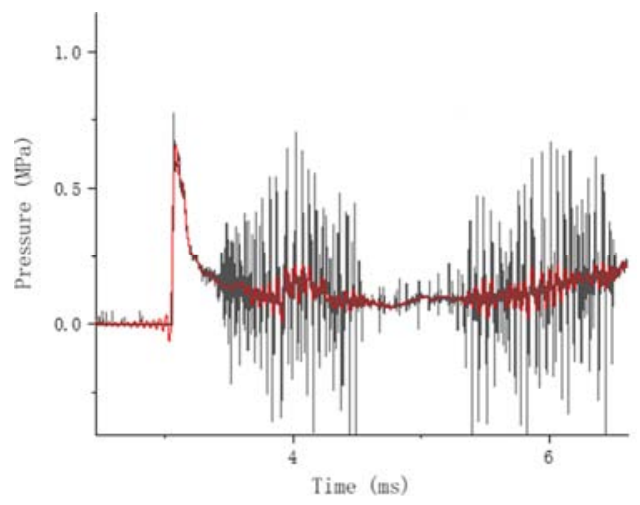

b）滤波后压力时程曲线图（红色线）

图4 测点3\#压力时程曲线图。 
对其余测点进行相同的数据处理, 得到如图5所示压 力时程曲线图。

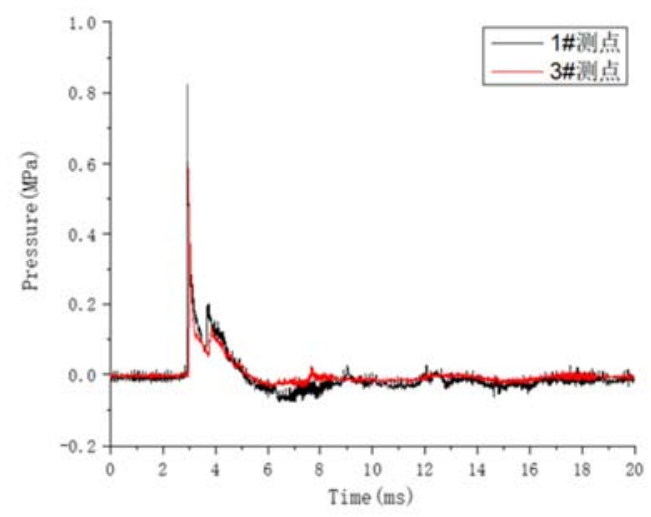

a) $1 \#$ 与 $3 \#$ 测点压力时程曲线

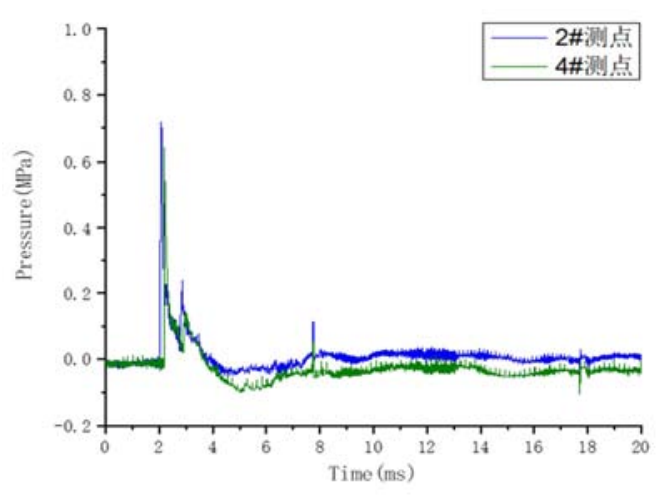

b) $2 \#$ 与 $4 \#$ 测点压力时程曲线

图5 各测点压力时程曲线。

根据图 $5 a$ )可得 $1 \#$ 与 $3 \#$ 两个纵向测点的初始作用时间 及超压峰值对比情况, 如表4所示。

表4 1\#与 3 \#测点压力时程曲线。

\begin{tabular}{llll}
\hline \multirow{2}{*}{ 参数 } & 测点 & & \multirow{2}{*}{ 差值 } \\
\cline { 2 - 3 } & $\mathbf{1 \#}$ & $\mathbf{3 \#}$ & 0.581 \\
\hline 冲击波作用初始时间 $/ \mathrm{ms}$ & 2.913 & 3.494 & 0.513 \\
超压峰值 $/ \mathrm{Mpa}$ & 0.812 & 0.634 & -0.178 \\
\hline
\end{tabular}

由表4可以看出, 两个纵向测点之间, 处于低位的 $1 \#$ 测点, 在爆炸发生之后, 其初始作用时间会早于位置较高 的3\#测点, 即冲击波波阵面会沿着抗爆墙由下至上传播; 从超压峰值的数据对比来看, 1 \#测点超压峰值大于 3 \#测点 超压峰值, 说明爆炸冲击波在沿墙面向上传播的过程中, 超压峰值会随着传播的过程而逐渐衰减。

根据图 $5 b$ ) 可得 $2 \#$ 与 $4 \#$ 两个横向测点的初始作用时间 及超压峰值对比情况, 如表5所示。

表5 2\#与4\#测点压力时程曲线。

\begin{tabular}{llll}
\hline 参数 & \multicolumn{2}{l}{ 测点 } & \multirow{2}{*}{ 差值 } \\
\cline { 2 - 3 } & $\mathbf{2 \#}$ & $\mathbf{4 \#}$ & \\
\hline 冲击波作用初始时间 $/ \mathrm{ms}$ & 2.951 & 3.129 & 0.178 \\
超压峰值 $/ \mathrm{Mpa}$ & 0.687 & 0.634 & -0.053 \\
\hline
\end{tabular}

由表 5 可以看出, 两个横向测点之间, 冲击波首先作 用在 2 \#测点, 这种情况出现是因为 2 \#测点处于炸药在墙面 投影点处; 超压峰值对比来看, 2 \#测点超压值大于 $4 \#$ 测点 超压值, 说明爆炸冲击波在沿着中心向两边传播过程中衰 减。

从各测点超压时程曲线的对比可以发现, 纵向高度不 同的测点对应的时程曲线相似, 因为相同工况下纵向高度 不同的测点, 主要变化的是爆炸源到测点之间的距离, 但 是测点对应的冲击波的变化规律不会产生太大的差异。在 相同工况下, 爆炸产生的冲击波总是最先到达纵向高度最 低的1井测点, 再依次向上传导。从测点产生的超压峰值大 小角度探讨, $1 \#$ 、2\#、3\#测点对应的超压峰值逐渐减小。

出现这种情况的原因是在爆炸过程中, 向下传播的冲 击波遇到地面反射, 与向前行进的冲击波耦合叠加, 形成 了速度更快能量更大的马赫波, 因而纵向高度最低的 $1 \#$ 测点最先受到冲击波影响, 之后是与爆源距离最近的2\# 测点, 而3\#测点距离爆源距离最远, 冲击波到达时间最长, 这说明了冲击波作用的先后受到爆心距与纵向高度的共 同影响。由于耦合产生的马赫波强度更大, 因而造成1\# 测点对应的超压峰值比 $2 \#$ 测点对应的超压峰值要大, $3 \#$ 测点与爆源距离最远, 冲击波衰减明显, 所以对应的超压 峰值最小。

读取各测点初始作用时间及超压值, 如表6所示, 为 验证数值仿真做准备。

表6 各测点初始作用时间与超压峰值。

\begin{tabular}{lllllllll}
\hline \multirow{2}{*}{ 工况 } & \multicolumn{9}{l}{ 冲击波作用初始时间/ms } \\
\cline { 2 - 9 } & $\mathbf{1 \#}$ & $\mathbf{2 \#}$ & $\mathbf{3 \#}$ & $\mathbf{4 \#}$ & $\mathbf{1 \#}$ & $\mathbf{2 \#}$ & $\mathbf{3 \#}$ & $\mathbf{4 \#}$ \\
\hline 试验数据 & 2.913 & 2.951 & 3.494 & 3.129 & 0.812 & 0.687 & 0.607 & 0.634 \\
\hline
\end{tabular}

\section{3. 数值模拟}

\section{1. 数值模拟耦合算法}

本文采用多物质ALE算法模拟爆炸场单元 (炸药及空 气), 采用Lagrangian网格模拟结构单元 (抗爆墙及地面), 通过流固耦合方式定义爆炸冲击波和结构的耦合作用。在 LS-DYNA 中, 流固耦合通过
*CONSTRAINED_LAGRANGE_ IN_SOLID 来实现 [10-12]。

其中, FRCMIN为耦合最小体积分数定义项, 本文研 究的爆炸冲击问题, 为有效地减小了爆炸流场穿透或泄露 现象, 计算中该值设置为 0.3 , 使得耦合计算较早发生。

ILEAK为耦合泄漏控制选项, 为更准确模拟爆炸冲击波与 结构的耦合作用, 该值设置为 2 , 即耦合泄露等级为强, 并且能够提供更精确的能量算法, 当流体穿透体积分 数 $>$ FRCMIN +0.4 时关闭耦合泄漏开关。 
3.2. 材料模型和状态方程

1) 混凝土采用

*MAT_JOHNSON_HOLMQUIST_CONCRETE材料模型 [13-14]

其强度按无量纲等效应力表示为:

$$
\sigma^{*}=\left[A(1-D)+B P^{* N}\right][1-c \ln (\dot{\varepsilon})]
$$

式中, $\sigma^{*}$ 为实际等效应力与准静态单轴抗压强度 $f_{c}$ ' 之比; $P^{*}$ 为无量纲压力; $\dot{\varepsilon}$ 为无量纲应变率; $A$ 为无量纲粘 聚强度, $B$ 为无量纲硬化系数; $c$ 为应变率系数; $N$ 为应变 硬化参数。混凝土的H-J-C本构模型参数取值, 如表 7 所示。

表7 混凝土的材料参数。

\begin{tabular}{cllllllllll}
\hline 参数 & $\boldsymbol{\rho}_{\mathbf{0}}$ & $\boldsymbol{G}$ & $\boldsymbol{A}$ & $\boldsymbol{B}$ & $\boldsymbol{c}$ & $\boldsymbol{N}$ & $\boldsymbol{f}_{\boldsymbol{c}} \boldsymbol{\prime}$ & $\boldsymbol{T}$ & $\boldsymbol{\varepsilon}_{\mathbf{0}}$ & $\boldsymbol{\varepsilon}_{\min }$ \\
\hline 取值 & 2400 & 14.86 & 0.79 & 1.6 & 0.007 & 0.61 & $48 \times 10^{6}$ & $4 \times 10^{6}$ & 0.001 & 0.01 \\
\hline
\end{tabular}

表7 继续。

\begin{tabular}{lllllllllll}
\hline 参数 & $\boldsymbol{S}_{\max }$ & $\boldsymbol{P}_{\boldsymbol{C}}$ & $\boldsymbol{\mu}_{\mathrm{C}}$ & $\boldsymbol{P}_{\text {lock }}$ & $\boldsymbol{\mu}_{\text {lock }}$ & $\boldsymbol{D}_{\mathbf{1}}$ & $\boldsymbol{D}_{\mathbf{2}}$ & $\boldsymbol{K}_{\mathbf{1}}$ & $\boldsymbol{K}_{\mathbf{2}}$ & $\boldsymbol{K}_{\mathbf{3}}$ \\
\hline 取值 & 7.0 & 16 & 0.001 & 0.8 & 0.1 & 0.04 & 1 & 85 & -171 & 208 \\
\hline
\end{tabular}

混凝土单元的破坏条件用关键字 *MAT_ADD_EROSION来定义, 设定混凝土的最大主拉应 变为 0.01 , 当主拉应变达到设定值时, 混凝土单元破坏失 效, 并从模型中消失。

2)钢筋采用*MAT_PLASTIC_KINEMATIC材料模型

钢筋的模型参数取值, 如表 8 所示 $[14]$ 。

表8 钢筋的材料参数。

\begin{tabular}{|c|c|c|c|c|c|c|c|c|}
\hline 参数 & $\rho_{0}$ & $E$ & $v$ & SIGY & ETAN & $C$ & $P$ & $\varepsilon$ \\
\hline 取值 & $7.85 \times 10^{3}$ & $2 \times 10^{11}$ & 0.3 & $4 \times 10^{8}$ & $2 \times 10^{9}$ & 40 & 5 & 0.1 \\
\hline
\end{tabular}

3)空气模型采用*MAT NULL材料模型

模 型 需使用线性多项式状态方程 *EOS_LINEAR_POLYNOMAL配合来描述空气[15]。其线 性方程表达式为:

$P=C_{0}+C_{1} \mu+C_{2} \mu^{2}+C_{3} \mu^{3}+\left(C_{4}+C_{5} \mu+C_{6} \mu^{2}\right) E_{0}$

其中, $\mu=\frac{\rho}{\rho_{0}}-1, P$ 为压力; $C_{0}-C_{6}$ 为实常数; $\mu$ 为体 积变化率; $E_{0}$ 为初始单位内能 $\left(\mathrm{MJ} \cdot \mathrm{m}^{-3}\right) ; \rho$ 为质量密度 $\left(\mathrm{kg} \cdot \mathrm{m}^{-3}\right) ; \rho_{0}$ 为参考质量密度 $\left(\mathrm{kg} \cdot \mathrm{m}^{-3}\right)$ 。参数按理想气 体状态方程取值，如表9所示， $V_{0}$ 表示初始相对体积。

表9 空气的材料参数。

\begin{tabular}{lllllllllll}
\hline 参数 & $\boldsymbol{\rho}_{\mathbf{0}}$ & $\mathbf{C}_{\mathbf{0}}$ & $\mathbf{C}_{\mathbf{1}}$ & $\mathbf{C}_{2}$ & $\mathbf{C}_{3}$ & $\mathbf{C}_{4}$ & $\mathbf{C}_{5}$ & $\mathbf{C}_{\mathbf{6}}$ & $\mathbf{E}$ & $\boldsymbol{V}_{\mathbf{0}}$ \\
\hline 取值 & 1.29 & 0 & 0 & 0 & 0 & 0.4 & 0.4 & 0 & 0.25 & 1.0 \\
\hline
\end{tabular}

4) 炸 药 模 型采用高能炸药 *MAT_HIGH_EXPLOSIVE_BURN材料模型[15]

炸药产生的爆轰气体采用JWL状态方程来进行描述, 表达式为:

$$
P=A\left(1-\frac{\omega}{R_{1} V}\right) e^{-R_{1} V}+B\left(1-\frac{\omega}{R_{2} V}\right) e^{-R_{2} V}+\frac{\omega E_{0}}{V}
$$

式中: $P$ 为压力; $A 、 R_{1} 、 R_{2} 、 \omega$ 为JWL状态方程参数, $E_{0}$ 为初始内能 $\left(\mathrm{GJ} \cdot \mathrm{m}^{-3}\right), V_{0}$ 表示初始相对体积, $D$ 表示炸 药的爆速 $\left(\mathrm{m} \cdot \mathrm{s}^{-1}\right), P_{\mathrm{CJ}}$ 表示爆压 $(\mathrm{GPa}), \rho$ 表示炸药密度 $\left(\mathrm{kg} \cdot \mathrm{m}^{-3}\right)$, 参数取值如表 10 。

表10 炸药的材料参数。

\begin{tabular}{cllllllllll}
\hline 参数 & $\boldsymbol{A}$ & $\boldsymbol{B}$ & $\boldsymbol{R}_{\mathbf{1}}$ & $\boldsymbol{R}_{\mathbf{2}}$ & $\boldsymbol{\omega}$ & $\boldsymbol{E}_{\mathbf{0}}$ & $\boldsymbol{V}_{\mathbf{0}}$ & $\boldsymbol{D}$ & $\boldsymbol{P}_{\mathrm{CJ}}$ & $\boldsymbol{\rho}$ \\
\hline 取值 & 317 & 3.23 & 4.15 & 0.95 & 0.3 & 7 & 1 & 6718 & 18.5 & 1630 \\
\hline
\end{tabular}

5)刚性地面选用*MAT RIGID材料模型

爆炸场地地面设定参数, 密度 $2380 \mathrm{~kg} / \mathrm{m}^{3}$, 弹性模量 $3 \times 10^{8} \mathrm{~Pa}$, 泊松比为 0.3 。参数CON1、CON2取 7 , 表示约 束 $x, y$ 和 $z$ 三个方向的位移和转角。

\section{3. 爆炸场工况}

近地爆炸数值模拟分析由空气、炸药、抗爆墙和地面 四部分组成, 本模型基于空气、炸药均为均匀介质的假定, 爆炸过程为绝热过程。采用全尺寸建模, 空气域尺寸取 $5 \mathrm{~m} \times 3 \mathrm{~m} \times 5 \mathrm{~m}$, 以地面为原点, 地面以上 $3 \mathrm{~m}$, 地面以下 $2 \mathrm{~m}$, 空气边界施加非反射边界条件。炸药药量 $1 \mathrm{~kg}$, 距离 地面 $0.47 \mathrm{~m}$, 起爆点为炸药中心, 0 时刻起爆, 计算时间 $20 \mathrm{~ms}$, 时间步长 0.67 。抗爆墙采用分离式建模, 钢筋与混凝土共 用节点。抗爆墙迎爆面尺寸如图1所示, 墙内钢筋选用 BEAM161单元, 空气、炸药、墙体及地面均选用SOLID164
单元进行模拟。混凝土墙体、地面使用拉格朗日网格, 墙 体和地面固连, 采用自动面面接触。空气、炸药和地面均 使用映射方法进行网格划分, 炸药和空气按 $0.05 \mathrm{~m} \times$ $0.05 \mathrm{~m} \times 0.05 \mathrm{~m}$ 进行网格划分, 混凝土墙与钢筋按 $0.025 \mathrm{~m} \times 0.025 \mathrm{~m} \times 0.025 \mathrm{~m}$ 进行网格划分。模型中炸药、 地面及抗爆墙相对位置关系如图6所示。

试验中对墙体迎爆面设置的超压测点, 其中2\#超压测 点处于炸药在墙面的投影点处。1\#超压测点位于 2 \#测点下 方 $0.135 \mathrm{~m}$ 处, 3 \#超压测点位于 $2 \#$ 测点上方 $0.215 \mathrm{~m}$ 处。根据 试验中所确定的测点, 按位置坐标索引出测点对应的单元 号, 如图3所示, 1\#测点对应315777单元, 2\#测点对应 315977 单元, 3\#测点对应316477单元, 从数值模拟结果文 件中提取出测点的超压数值。 

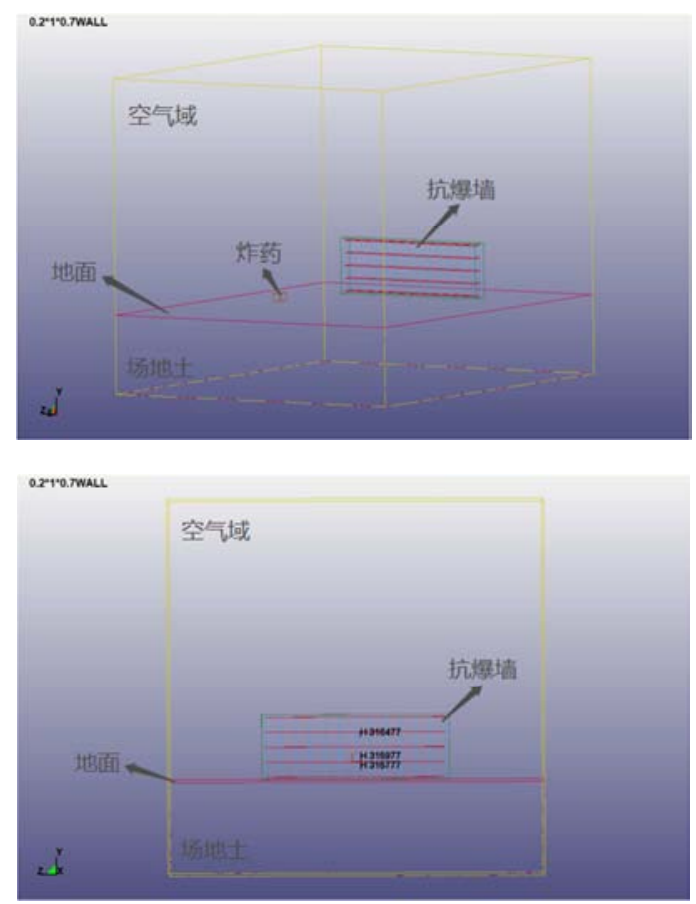

图6 近地面爆炸场与钢筋混凝土耦合模型示意图。

\section{4. 耦合模型数值模拟结果}

$1 \#$ 测点和 $3 \#$ 测点的数值模拟超压时程曲线如图7所示。

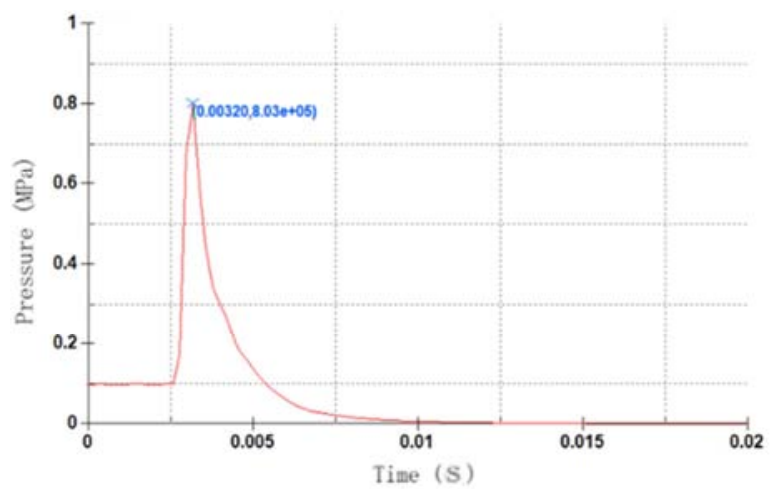

(a) 1 \#测点

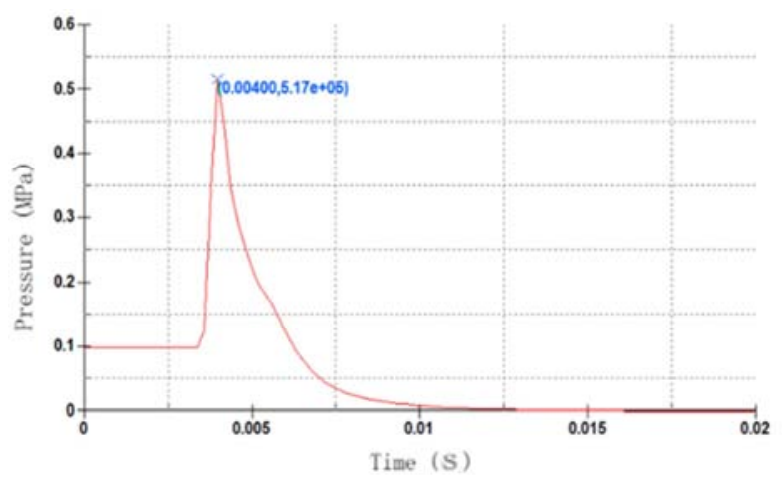

(b) 3 \#测点

图7 试验测点数值模拟压力时程曲线。
根据后处理软件可获取测点处超压时程曲线, 表 11 给出了 $1 \# 、 2 \# 、 3 \#$ 测点试验数据与数值模拟的对比情况。

表11 试验与仿真数据对比。

\begin{tabular}{|c|c|c|c|c|c|c|}
\hline \multirow{2}{*}{ 工况 } & \multicolumn{3}{|c|}{ 冲击波作用初始时间/ms } & \multicolumn{3}{|c|}{ 超压峰值/Mpa } \\
\hline & $1 \#$ & $2 \#$ & $3 \#$ & $1 \#$ & $2 \#$ & $3 \#$ \\
\hline 试验数据 & 2.913 & 2.951 & 3.494 & 0.812 & 0.687 & 0.607 \\
\hline 数值模拟 & 3.201 & 3.221 & 4.030 & 0.803 & 0.581 & 0.517 \\
\hline 相对误差 & $9.89 \%$ & $9.15 \%$ & $15.34 \%$ & $-1.11 \%$ & $-15.43 \%$ & $-14.83 \%$ \\
\hline
\end{tabular}

根据表 11 可知, 试验结果与数值模拟结果, 相同工况 下的初始作用时间, 近地面爆炸产生的冲击波往往最先传 播至纵向高度处于低位的1\#测点, 之后慢慢升高, 依次到 达2\#测点和 $3 \#$ 测点。从超压峰值的角度来分析, 1\#、2\#、 3\#测点的超压峰值随着纵向高度的上升而逐渐减小。

纵向对比来看, 试验数据比数值模拟的数值之间存在 误差, 爆炸过程中的周边环境影响是导致误差产生的最主 要原因, 冲击波受周边的墙壁反射冲击波的耦合叠加形成 了速度更快能量更大的马赫波, 因而无论是超压峰值还是 冲击波作用的初始时间都会与数值模拟情况有所不同。但 是在爆炸冲击波的数值计算与试验数据的对比的误差率 在可接受的范围内, 而且整个爆炸冲击波的变化规律也实 际情况相同。因此, 近地面爆炸场与钢筋混凝土抗爆墙的 耦合模型可以按本文的参数设置来进行模拟。

\section{4. 结论}

本文通过ANSYS/LS-DYNA软件建立模型, 并通过与 试验数据对比, 对所建模型的准确性进行验证, 获得了合 理的建模方式和材料参数取值, 为抗爆墙与爆炸场的耦合 模型建模提供了一种手段。

(1)对相似理论设计的抗爆墙进行试验, 采集了在冲击 波荷载作用下迎爆面处四个测点的超压变化情况, 通过数 据处理得到了其对应的超压时程曲线, 分析数据可知, 在 相同工况下, 爆炸产生的冲击波总是最先到达纵向高度最 低的点, 再依次向上传导。从测点产生的超压峰值大小角 度来讲, 超压峰值随着高度增大而逐渐减小。

(2)钢筋混凝土抗爆墙结构的模型采用分离式方法建 模, 结合自由空气爆炸场, 建立了近地面爆炸场与钢筋混 凝土墙的耦合模型。将数值计算的结果与试验测量所得数 据进行对比, 结果符合度较高, 为钢筋混凝土与爆炸场耦 合模型的建模方式提供了依据。

\section{参考文献}

[1] J.Henrych著,熊建国等译.爆炸动力学及其应用 [M].北京:科 学出版社, 1979.

[2] 张守中,孙业斌.爆炸载荷作用下刚塑性圆柱壳体的变形和 破裂 $[\mathrm{J}]$.兵工学报,1985(02):59-65.

[3] Zhang Shouzhong,Sun Yebin. Deformation and rupture of rigid-plastic cylinder shell due to explosion[J]. Acta Armamentarii, 1985(02):59-65. 
[4] Wu Chengqing, Hao Hong. Modeling of simultaneous ground shock and airblast pressure on nearby structures from surface explosions[J].International Journal of Impact Engineering, 2005,31(6):699-717.

[5] 仲倩,王伯良,黄菊,等.TNT空中爆炸超压的相似律 [J].火炸 药学报,2008,33(4):32-35.

[6] ZHONG Qian,WANG Bo-liang.HU ANG Ju, HUI Jun-ming. Study on the Similarity Law of TNT Explosion Overpressure in Air[J]. Chinese Journal of Explosives \& Propellants, 2008,33(4):32-35.

[7] 梁利平,李金擈.挡墙对爆炸冲击波传播影响的三维数值模拟 [J].福建师范大学福清分校学报,2011(05):26-30.

[8] LIANG Liping,LI Xin. Three- dimensional Numerical Value Simulation of Retailing Wall to the Blast Wave Propagating Influence[J].Journal of Fuqing branch of Fujian normal university, 2011(05):26-30.

[9] 刘伟,郑毅,秦飞.近地面TNT爆炸的试验研究和数值模拟 [J]. 爆破,2012(03):1-9.

[10] LIU Wei,ZHENG Yi,QIN Fei. Experimental and Numerical Simulation of TNT Explosion on the Ground $[\mathrm{J}]$. BLASTING,2012(03):1-9.

[11] 白金泽.LS-DYNA3D理论基础与实例分析[M].北京:科学出 版社,2005.

[12] BAI jinze. Theoretical basis and case analysis of LS-DYNA3D[M].beijing: Science Press, 2005.

[13] 史春芳,徐赵东.工程结构抗爆技术的研究现状[J].西安建筑 科技大学学报(自然科学版),2007(05):616-620.

[14] SHI Chun-fang,XU Zhao-dong.Anti-explosion technology in engineering structure[J]. Xi'an Univ.of Arch.\&Tech.(Natural Science Edition),2007(05):616-620.

[15] 杨俊杰.相似理论与结构模型试验 $[\mathrm{M}]$.武汉:武汉理工大学 出版社.2005.
[16] LS-DYNA Keyword User's Manual(LS-DYNA 用户 手 册) $[\mathrm{M}] .2017$.

[17] Alia A,Souli M,High explosive simulation using multi-material formulations[J]. Applied Thermal Engineering,2006,26(10):1032-1042.

[18] 李楠.钢纤维高强混凝土墙的抗爆性能有限元分析[D]. 长安 大学,2012.

[19] Li Nan.Finite Element Anslysis of Steel Fiber Reinforced High Strength Concrete Walls Subjected to Blasting Load.[D].Chang'an University,2012.

[20] 穆朝民,任辉启,李永池, 等.爆炸冲击波作用于墙体及对墙体 绕射的实验研究 [J].实验力学, 2008,23(02): 169-174.

[21] M U Chao-min, REN Hui-qi, LI Yong-chi, XIN Kai. Experimental Study of Blast Wave Reflection and Diffraction on a Shelter Wall[J]. Journal of Experimental Mechanics, 2008,23(02): 169-174.

[22] 张千里,张耀,年金哲.混凝土防爆墙对爆炸冲击波传播的影 响[J].振动与冲击, 2013(24): 192-197.

[23] ZHANG Qian-li,ZHANG Yao,NIAN Xin-zhei. Effect of concrete protective wall on explosion shock wave[J]. Journal of Vibration and Shock,2013(24): 192-197.

[24] 陈金, 高轩能. 炸药近地爆炸的数值模拟及影响参数的分析 [J].华侨大学学报(自然科学版),2014,35(05):570-575.

[25] Chen Xin, Gao Xuanneng,Numerical Simulation and Analysis of Influence Paramenters for Explosions Near Ground[J].Journal of Huaqiao University(Natural Science),2014,35(05):570-575.

\section{作者简介}

朱洤(1993-), 男, 硕士生, 主要研究方向: 工程防护, E-mail:783148731@qq.com 